\title{
Acute pancreatitis associated with indigo naturalis in pediatric severe Crohn's disease
}

\author{
Hyeon-A Kim, Hyo-rim Suh, Ben Kang, Byung-Ho Choe, on behalf of Crohn's and Colitis Association in \\ Daegu-Gyeongbuk (CCAiD) \\ Department of Pediatrics, School of Medicine, Kyungpook National University, Daegu, Korea
}

The incidence of inflammatory bowel disease (IBD) is rapidly increasing worldwide. Indigo naturalis is known to have an antiinflammatory effect. Indigo naturalis has been traditionally used in the treatment of IBD in China and Japan. Currently, it is used as a primary or adjunctive drug in patients with ulcerative colitis. There are some reports of the effects of indigo naturalis when used in patients with ulcerative colitis. However, its usage has been associated with adverse events, including liver dysfunction, headache, gastrointestinal disturbance, and pulmonary hypertension. Pancreatitis as an adverse event during treatment using indigo naturalis has not yet been reported. We report a case of recurrent events of pancreatitis that occurred briefly after starting medication with indigo naturalis in a child with severe Crohn's disease. The pancreatitis improved after indigo naturalis was discontinued in 2 events. This is the first case to report the association between pancreatitis and indigo naturalis in the English literature. (Intest Res 2019;17:144-148)

Key Words: Crohn disease; Inflammatory bowel disease; Pancreatitis; Child; Kampo

\section{INTRODUCTION}

The incidence of IBD in children is rapidly increasing worldwide, especially in Asia. ${ }^{1}$ Approximately $30 \%$ of patients develop the disease before 20 years of age. ${ }^{2}$ In children and adolescents, CD may present with distinct features such as poor growth and delayed sexual maturation. ${ }^{3}$ Current treatment options for pediatric CD include 5-aminosalicylic acid (5-ASA); corticosteroids; immunosuppressants such as azathioprine (AZA), 6-mercaptopurine, or methotrexate; exclusive enteric nutrition; and biologics. ${ }^{4}$ Anti-TNF agents, such as infliximab and adalimumab, are recommended as a second-line therapy

Received July 10, 2018. Revised August 1, 2018. Accepted August 11, 2018 Correspondence to Byung-Ho Choe, Department of Pediatrics, School of Medicine, Kyungpook National University, 130 Dongdeok-ro, Jung-gu, Daegu 41944, Korea. Tel: +82-53-200-5704, Fax: +82-53-425-6683, E-mail: bhchoe@knu.ac.kr

ORCID Hyeon-A Kim (https://orcid.org/0000-0001-9843-0188), Byung-Ho Choe (https://orcid.org/0000-0001-9899-9120) for pediatric patients with severe active $\mathrm{CD}$ following failure of or intolerance to conventional therapies, although recent treatment strategies have evolved into introducing them earlier in the course of the disease. ${ }^{5,6}$

Indigo naturalis is known to have an anti-inflammatory effect, and it has been used in the treatment of psoriasis, chronic hemorrhagic radiation proctitis, and IBD in Japan. However, adverse events, including liver dysfunction, headache, GI disturbance, and pulmonary hypertension, have been reported with its use. ${ }^{7.8}$

Here, we report the first case of acute pancreatitis associated with indigo naturalis in a child with refractory CD.

\section{CASE REPORT}

An 11-year-old boy was admitted to Kyungpook National University Children's Hospital owing to abdominal pain, diarrhea, and hematochezia for 3 months. A weight loss of $3 \mathrm{~kg}$, without 
any decrease in growth velocity, was observed. He underwent incision and drainage for a perianal fistula, 6 months before admission. On admission, his vital signs were stable and within normal limits. An opened perianal fistula was observed at the 11 o'clock position beneath the right scrotum (Fig. 1).

Initial laboratory tests showed the following results: white blood cell (WBC) count, 5,060/ $\mu \mathrm{L}$; hemoglobin level, $12.3 \mathrm{~g} /$ dL; platelet count, 352,000/ $\mu \mathrm{L}$; albumin level, $4.4 \mathrm{~g} / \mathrm{dL}$; ESR, 3 $\mathrm{mm} / \mathrm{hr}$; CRP level, $0.01 \mathrm{mg} / \mathrm{dL}$. Stool occult blood test was positive, and fecal calprotectin level was $>2,000 \mu \mathrm{g} / \mathrm{g}$. No pathogens were observed on stool culture and stool PCR. Ileocolonoscopy showed small ulcers from the terminal ileum to the descending colon with continuous mucosal swelling, erythema, partial vascularity loss, and friability, favoring a diagnosis

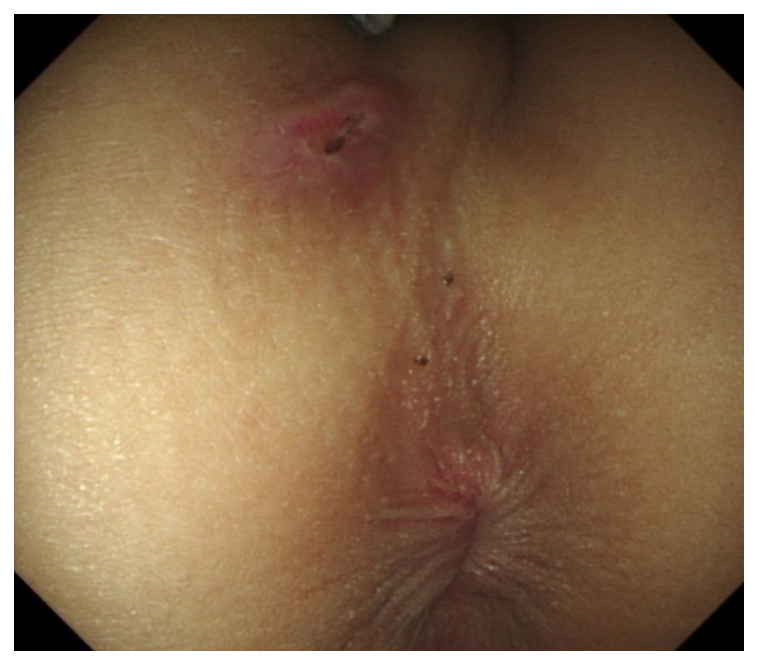

Fig. 1. Initial gross examination. Anal fistula opening site at the 11 o'clock position without discharge. of UC with backwash ileitis (Fig. 2). Upper GI endoscopy revealed erosions in the stomach. Histologic examination of the biopsy samples taken during the endoscopic examinations revealed non-caseating granulomas in the cecum. Magnetic resonance enterography detected no involvement of the small bowel (Fig. 3). The patient was diagnosed with CD based on the findings of non-caseating granuloma showing a phenotype of Alb, L3+ L4a, B1p, G0 according to Paris classification. His initial Pediatric CDAI (PCDAI) score was 55.

Initial treatment was started with exclusive enteral nutrition, mesalazine, and AZA. However, the symptoms of the patient failed to improve. Despite treatment with prednisolone (1 mg/ $\mathrm{kg} /$ day) for 2 weeks, the symptoms of the patient persisted. Subsequently, infliximab was added to the previous medication; after 2 doses of infliximab, the PCDAI decreased to 25. However, his symptoms aggravated 5 weeks later when corticosteroids were tapered to $0.25 \mathrm{mg} / \mathrm{kg} /$ day. The patient had a PCDAI score of 60 and was readmitted for re-evaluation.

Ileocolonoscopy showed no improvement, and PCR of the biopsied samples showed negative results for cytomegalovirus. Laboratory examination showed the following results: ESR, $21 \mathrm{~mm} / \mathrm{hr}$; CRP level, $2.2 \mathrm{mg} / \mathrm{dL}$; amylase level, $41 \mathrm{U} / \mathrm{L}$; and lipase level, $39 \mathrm{U} / \mathrm{L}$. Stool occult blood test was positive, and fecal calprotectin level was $1,525 \mu \mathrm{g} / \mathrm{g}$. No pathogens were detected on stool culture or stool bacterial PCR. Treatment with intravenous prednisolone at doses of $1.5 \mathrm{mg} / \mathrm{kg} / \mathrm{day}$ was started and infliximab infusion was shortened to 4-week intervals. Oral mesalazine and AZA were discontinued owing to GI discomfort, and low-dose oral methotrexate at $15 \mathrm{mg} / \mathrm{wk}$ was started.

Two months later, the patient relapsed while being treated
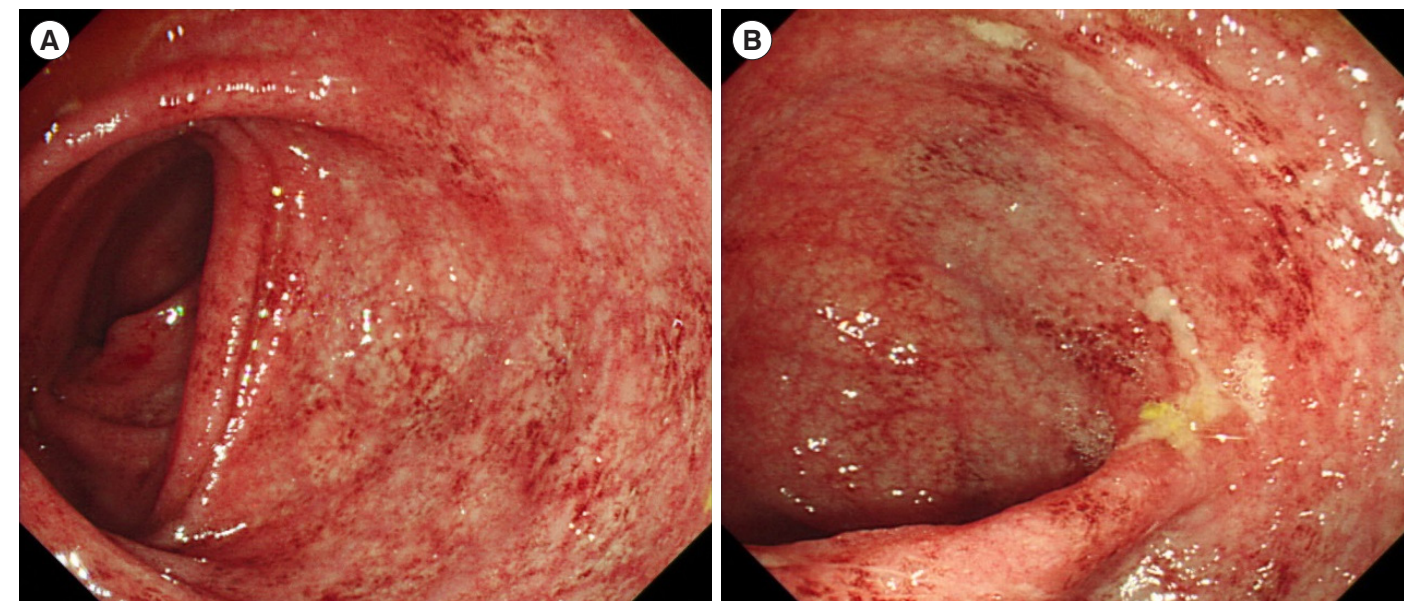

Fig. 2. Initial colonoscopic images. (A) Mucosal erythema with low vascularity is noted in the descending colon and (B) multiple ulcers are noted in the entire colon. 
with tapering oral prednisolone and was readmitted. His PCDAI score was 35, and follow-up sigmoidoscopy and blood tests provided nonspecific findings except the following results: hemoglobin level, $9.8 \mathrm{~g} / \mathrm{dL}$; ESR, $58 \mathrm{~mm} / \mathrm{hr}$; CRP level, $0.03 \mathrm{mg} /$ $\mathrm{dL}$; fecal calprotectin level $>2,000 \mu \mathrm{g} / \mathrm{g}$. As long-term corticosteroid use could be associated with severe adverse events, we decided to use indigo naturalis considering that the colon of the patient showed a UC-like appearance on endoscopy.

Indigo naturalis was added to the treatment at initial doses of $0.3 \mathrm{~g}$ /day per oral. However, after only 2 doses of indigo nat- uralis, the patient experienced epigastric pain and vomiting. Laboratory examination showed the following results: WBC count, 8,540/ $\mu \mathrm{L}$; hemoglobin level, $9.2 \mathrm{~g} / \mathrm{dL}$; platelet count, 379,000/ $\mu \mathrm{L}$; albumin level, $4.0 \mathrm{~g} / \mathrm{dL}$; ESR, $32 \mathrm{~mm} / \mathrm{hr}$; CRP level, $0.05 \mathrm{mg} / \mathrm{dL}$; amylase level, $199 \mathrm{U} / \mathrm{L}$; and lipase level, $476 \mathrm{U} /$ L. Indigo naturalis was discontinued, and the patient was put under fasting. Symptoms subsided after 2 days, and amylase and lipase levels normalized to $42 \mathrm{U} / \mathrm{L}$ and $46 \mathrm{U} / \mathrm{L}$, respectively, after 1 week. Oral tacrolimus was started at doses of 2 $\mathrm{mg} /$ day.
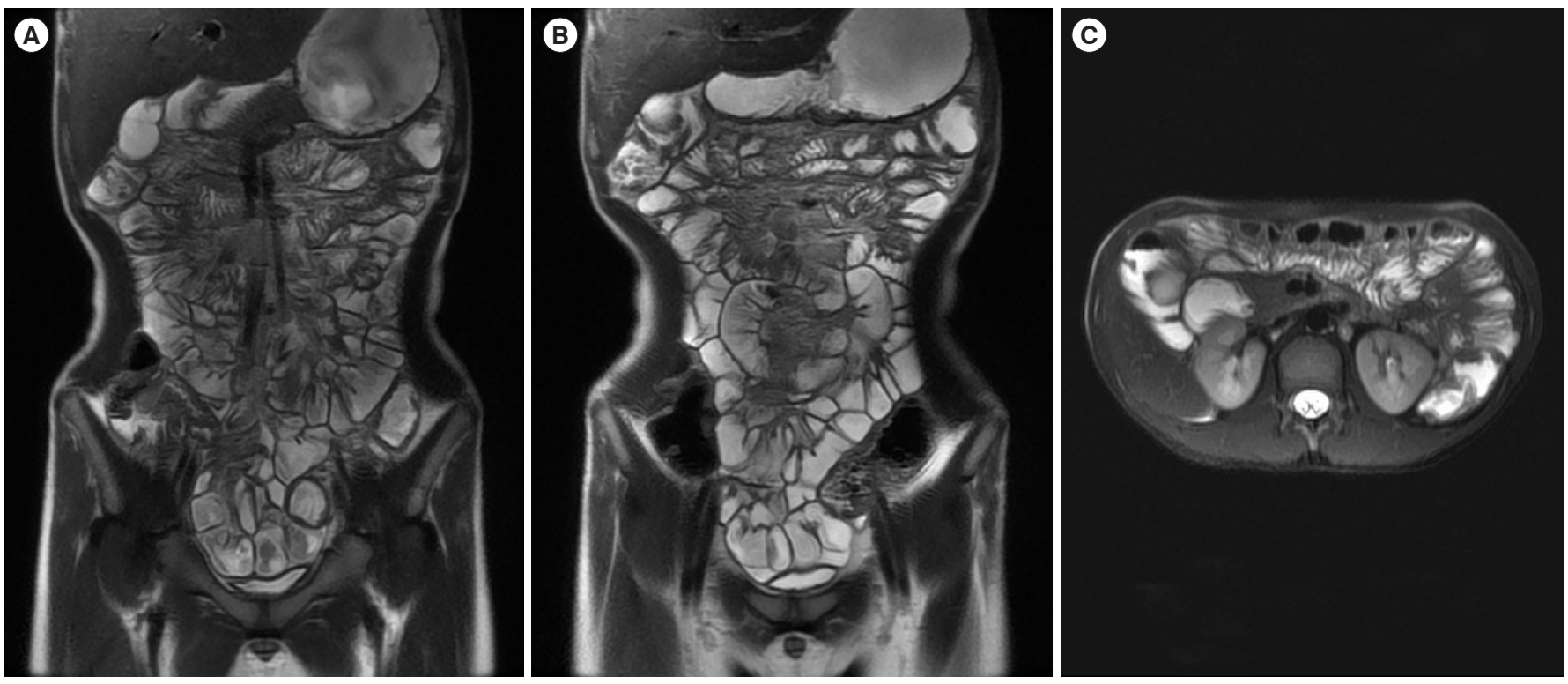

Fig. 3. Initial magnetic resonance enterography images. Wall thickening is observed in the entire colon, especially the descending colon and transverse colon. (A, C) Mild wall thickening of descending colon, (B) mild wall thickening of descending colon and pericolic fat infiltration.
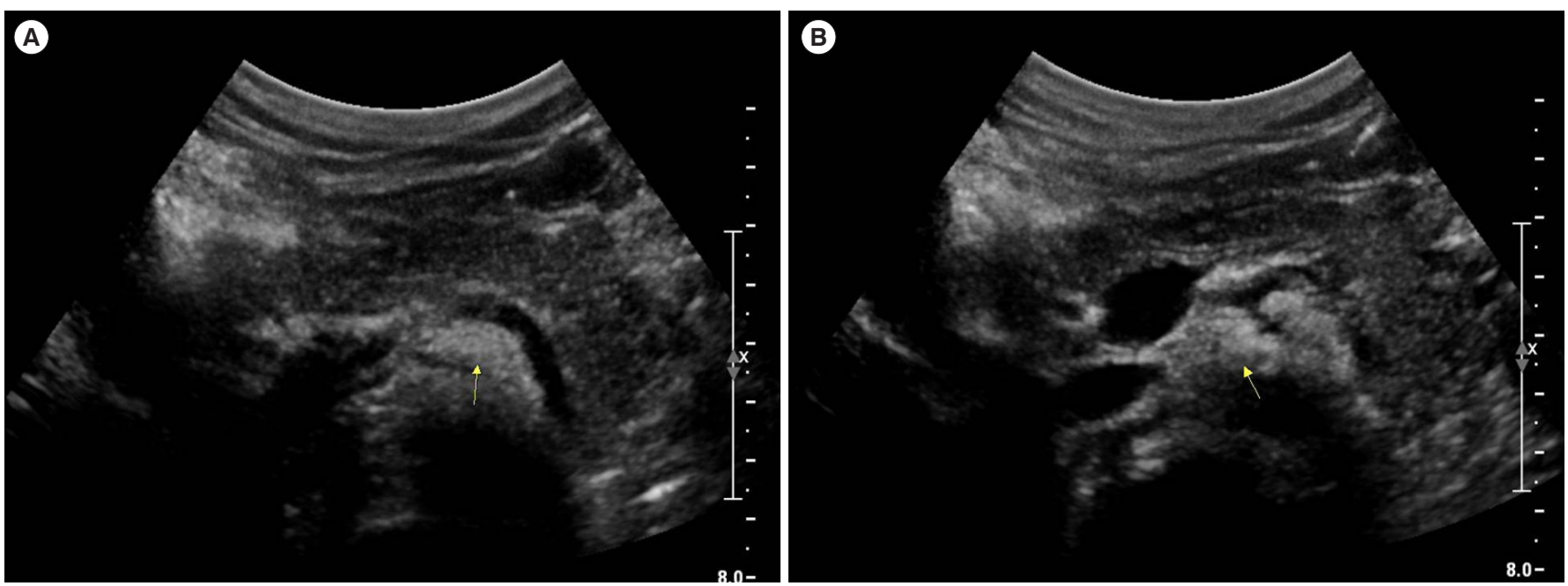

Fig. 4. Initial abdominal ultrasonography images. Suspected mild swelling is observed in the pancreas with peripancreatic fat infiltration (arrow in $A$ and $B$ ). 
Treatment with oral tacrolimus was not effective for 1 month, and we decided to try indigo naturalis with lower starting doses of $0.15 \mathrm{mg} /$ day. However, after 2 doses of indigo naturalis, the patient again experienced epigastric pain and vomiting. Laboratory exams showed the following results: WBC count, 11,150/ $\mu \mathrm{L}$; hemoglobin level, $10.8 \mathrm{~g} / \mathrm{dL}$; platelet count, 400,000/ $\mu \mathrm{L}$; albumin level, $3.6 \mathrm{~g} / \mathrm{dL}$; ESR, $36 \mathrm{~mm} / \mathrm{hr}$; CRP level, 0.12 $\mathrm{mg} / \mathrm{dL}$; amylase level, $144 \mathrm{U} / \mathrm{L}$; and lipase level, $345 \mathrm{U} / \mathrm{L}$. The peripancreatic fluid collection or fat stranding was not very evident by abdominal ultrasonogram, so that acute pancreatitis could be diagnosed by clinical correlation (Fig. 4). Subsequently, indigo naturalis was discontinued, and the patient was kept fasting. His symptoms subsided after 3 days, and his amylase and lipase levels normalized to $50 \mathrm{U} / \mathrm{L}$ and $43 \mathrm{U} / \mathrm{L}$, respectively, after 10 days. Written informed consent was obtained from the patient and his parents.

\section{DISCUSSION}

Indigo naturalis (Qing-Dai) has been traditionally used in the treatment of various inflammatory diseases and psoriasis in China and Japan. ${ }^{9}$ It is also used in various diseases such as ulcers in the mouth, chronic myeloid leukemia, and ulcerative proctitis. ${ }^{10,11}$ Currently, it is mainly used in the treatment of UC alone or as an adjuvant drug. ${ }^{12}$ There are some reports of the medicinal effects of indigo naturalis when used in patients with refractory UC. ${ }^{13}$ Xilei-san (a component of Indigo naturalis) enema made using Xilei-san, known as a compound of indigo naturalis, has been shown to be as effective as dexamethasone enema in mild-to-moderate ulcerative proctitis, showing a significant improvement in clinical, endoscopic, and histologic scores. ${ }^{14,15}$

Treatment of IBD with indigo naturalis has recently been reported, and studies on its efficacy have been carried out. There have been reports of the clinical efficacy and safety of Qing-Dai enema for the treatment of chronic hemorrhagic radiation proctitis. ${ }^{16}$ Another report states that Qing-Dai is clinically and endoscopically effective in patients with UC. ${ }^{13}$ In addition, it has been reported that the oral capsule Qing-Dai is clinically and endoscopically effective in patients with UC when treated for 8 weeks, and is effective in inducing remission in patients with moderate UC activity. ${ }^{17}$ Indigo naturalis has been tried in adults and was proven to be effective at a doses of 0.5 to $2 \mathrm{~g}$ /day without major side effects. ${ }^{7,17}$

The mechanism of indigo naturalis is mediated by its indigo molecule that acts like a human aryl hydrocarbon receptor
(AhR) ligand. ${ }^{12}$ The AhR ligand plays an important role in generating AhR-expressing type 3 innate lymphoid cells, which produce interleukin 22 (IL-22) in the gut mucosa. IL-22 stimulates the expression of tight junction molecules, which can enhance epithelial permeability and produce antimicrobial peptides. In addition, IL-22 induces signal transducer and activator of transcription 3 (STAT3) phosphorylation, which plays an important role in IL-22-dependent epithelial regeneration as well as organoid growth. Through these mechanisms, IL-22 contributes to the homeostasis of the intestinal immune systems and healing of the gut mucosa. ${ }^{12}$

Adverse events, such as pulmonary hypertension, liver dysfunction, GI disturbances, and headaches, have been reported with the use of indigo naturalis medication ${ }^{7,18}$ However, to date, there are no reports of pancreatitis as an adverse event during treatment using indigo naturalis. In the present case, pancreatitis occurred briefly after the addition of indigo naturalis, without any changes in previous medications. The pancreatitis improved after indigo naturalis was discontinued. This was observed twice in this patient, confirming the association of indigo naturalis and pancreatitis in this case.

In a study of patients with $\mathrm{CD}$, the causes of pancreatitis were gallstone (21\%), purine drug (6-mercaptopurine, AZA) (13\%), duodenal involvement of $\mathrm{CD}(12 \%)$, and other drugs (mesalamine, sulfasalazine, and 5-ASA) (4\%). ${ }^{19}$ Although the mechanism underlying the development of pancreatitis is not entirely clear, pancreatitis associated with AZA, 5-ASA, and sulfasalazine is dose independent and is thought to occur through hypersensitivity reactions. ${ }^{20}$ Considering that pancreatitis occurred just after 2 doses of indigo naturalis, a similar hypersensitivity reaction may have caused the pancreatitis in the current case.

In conclusion, we report a case of acute pancreatitis that was associated with treatment using indigo naturalis. To the best of our knowledge, this is the first case to report the association between pancreatitis and indigo naturalis in the English literature.

\section{FINANCIAL SUPPORT}

The authors received no financial support for the research, authorship, and/or publication of this article.

\section{CONFLICT OF INTEREST}

No potential conflict of interest relevant to this article was reported. 


\section{AUTHOR CONTRIBUTION}

Choe BH designed the report; Kim HA collected the patient's clinical data and wrote the paper; Suh H, Kang B, and Choe BH analyzed the data and edited

\section{REFERENCES}

1. Hong SJ, Cho SM, Choe BH, et al. Characteristics and incidence trends for pediatric inflammatory bowel disease in Daegu-Kyungpook Province in Korea: a multi-center study. J Korean Med Sci 2018;33:e132.

2. Lee YA, Chun P, Hwang EH, Mun SW, Lee YJ, Park JH. Clinical features and extraintestinal manifestations of Crohn disease in children. Pediatr Gastroenterol Hepatol Nutr 2016;19:236242.

3. Choi J, Kang B, Kim MJ, Sohn I, Lee HJ, Choe YH. Early infliximab yields superior long-term effects on linear growth in pediatric Crohn's disease patients. Gut Liver 2018;12:255-262.

4. Ruemmele FM, Veres G, Kolho KL, et al. Consensus guidelines of ECCO/ESPGHAN on the medical management of pediatric Crohn's disease. J Crohns Colitis 2014;8:1179-1207.

5. Kang B, Choe YH. Early biologic treatment in pediatric Crohn's disease: catching the therapeutic window of opportunity in early disease by treat-to-target. Pediatr Gastroenterol Hepatol Nutr 2018; 21:1-11.

6. Kang B, Choi SY, Kim HS, Kim K, Lee YM, Choe YH. Mucosal healing in paediatric patients with moderate-to-severe luminal Crohn's disease under combined immunosuppression: escalation versus early treatment. J Crohns Colitis 2016;10: 1279-1286.

7. Naganuma M, Sugimoto S, Mitsuyama K, et al. Efficacy of indigo naturalis in a multicenter randomized controlled trial of patients with ulcerative colitis. Gastroenterology 2018;154: 935-947.

8. Ye BD. Introducing traditional herbal medicine into conventional health care in treating ulcerative colitis: primum non nocere. Gastroenterology 2018;154:792-795.

9. Chang HN, Pang JH, Yang SH, et al. Inhibitory effect of indigo naturalis on tumor necrosis factor-alpha-induced vascular cell adhesion molecule-1 expression in human umbilical vein endothelial cells. Molecules 2010;15:6423-6435.

10. Fukunaga K, Hida N, Ohnishi K, et al. A suppository Chinese medicine (xilei-san) for refractory ulcerative proctitis: a pilot clinical trial. Digestion 2007;75:146-147.

11. Stasiak N, Kukuła-Koch W, Głowniak K. Modern industrial and pharmacological applications of indigo dye and its derivatives: a review. Acta Pol Pharm 2014;71:215-221.

12. Sugimoto S, Naganuma M, Kanai T. Indole compounds may be promising medicines for ulcerative colitis. J Gastroenterol 2016; 51:853-861.

13. Suzuki H, Kaneko T, Mizokami Y, et al. Therapeutic efficacy of the Qing Dai in patients with intractable ulcerative colitis. World J Gastroenterol 2013;19:2718-2722.

14. Zhang F, Li Y, Xu F, Chu Y, Zhao W. Comparison of Xilei-san, a Chinese herbal medicine, and dexamethasone in mild/moderate ulcerative proctitis: a double-blind randomized clinical trial. J Altern Complement Med 2013;19:838-842.

15. Ng SC, Lam YT, Tsoi KK, Chan FK, Sung JJ, Wu JC. Systematic review: the efficacy of herbal therapy in inflammatory bowel disease. Aliment Pharmacol Ther 2013;38:854-863.

16. Yuan G, Ke Q, Su X, Yang J, Xu X. Qing Dai, a traditional Chinese medicine for the treatment of chronic hemorrhagic radiation proctitis. Chin Ger J Clin Oncol 2009;8:114.

17. Sugimoto S, Naganuma M, Kiyohara H, et al. Clinical efficacy and safety of oral Qing-Dai in patients with ulcerative colitis: a single-center open-label prospective study. Digestion 2016;93: 193-201.

18. Nishio M, Hirooka K, Doi Y. Chinese herbal drug natural indigo may cause pulmonary artery hypertension. Eur Heart J 2016;37:1992

19. Moolsintong P, Loftus EV Jr, Chari ST, Egan LJ, Tremaine WJ, Sandborn WJ. Acute pancreatitis in patients with Crohn's disease: clinical features and outcomes. Inflamm Bowel Dis 2005; 11:1080-1084.

20. Gallego-Gutiérrez S, Navas-López VM, Kolorz M, et al. Successful mercaptopurine usage despite azathioprine-induced pancreatitis in paediatric Crohn's disease. J Crohns Colitis 2015;9:676-679. 